\title{
Miniaturized Fully-Passive Wireless Neural Recording with Heterogeneous Integration in Thin Packages
}

This paper was downloaded from TechRxiv (https://www.techrxiv.org).

\section{LICENSE}

CC BY 4.0

SUBMISSION DATE / POSTED DATE

31-01-2022 / 03-02-2022

\section{CITATION}

Been Sayeed, Sk Yeahia; Pulugartha, Markondeyaraj (2022): Miniaturized Fully-Passive Wireless Neural Recording with Heterogeneous Integration in Thin Packages. TechRxiv. Preprint. https://doi.org/10.36227/techrxiv.19093637.v1

$\mathrm{DOI}$

10.36227/techrxiv.19093637.v1 


\title{
Miniaturized Fully-Passive Wireless Neural Recording with Heterogeneous Integration in Thin Packages
}

\author{
Sk Yeahia Been Sayeed ${ }^{1}$, Satheesh B Venkatakrishnan ${ }^{2}$, John L. Volakis ${ }^{2}$ and Pulugurtha Markondeya \\ $\operatorname{Raj}^{1,2}$ \\ ${ }^{1}$ Dept. of Biomedical Engineering \\ ${ }^{2}$ Dept. of Electrical and Computer Engineering, \\ College of Engineering and Computing \\ Florida International University, Miami, FL 33174, USA \\ Email: sbeen002@fiu.edu
}

\begin{abstract}
Wireless fully-passive neural recording systems are demonstrated with heterogeneous integration of sensing, mixing and communication components in flexible polymer dielectric films. The recording neurosensor has an antenna, antiparallel diode, and a by-pass capacitor to modulate an incoming carrier signal with the neuropotentials and backscatter the mixed signal to an external reader circuit. Planar antennas are realized on Liquid Crystal Polymer (LCP) flexible substrates with low dielectric constant. The designed antenna topology is realized with a single metal layer and eliminates the dependency on substrate thickness and leads to thinner sensors. This is a major advance compared to prior passive neural recording with rigid and thicker substrates and components. Approximately $\mathbf{8 0 \%}$ thickness reduction and $20 \%$ volume reduction are achieved with similar performance compared to earlier studies. Our approach thus leads to lowcost and disposable wireless neurosensors on a flexible platform with heterogenous integration. Keywords: Heterogenous integration, Wireless Neural Recording, Liquid crystal polymer (LCP), wireless fully-passive neurosensor, miniaturization.
\end{abstract}

\section{INTRODUCTION}

Ever increasing demand for medical devices has been fueling vital innovations in package integration of flexible bioelectronics for diagnostic and therapeutic systems. Medical device packaging can be classified into multiple categories depending on their use in either implantable or on-skin wearable environments. Rigid components on thick circuit boards in hermetic packages with feedthroughs and connectors are most common for implantable medical systems. Encapsulated rigid packaged devices on flexible carriers, embedding thin bare chips in flexible substrates, fabric-integrated electronics, transferrable electronics as onskin e-tattoos, form the key available classes for wearable electronics. In all these classes of medical device packaging, there is a continual paradigm shift from rigid packages to thin flexible packages while still meeting the demand for seamless power and data connectivity, and sensor integration for health monitoring. This shift is to enhance user's comfort in terms of skin conformity, minimal obtrusiveness and invasiveness and also avoid the need for frequent replacement. Additionally, in order to facilitate easier surgical implantation and less inflammation in the human body, miniaturized flexible packaged devices are preferred for implantable devices. In order to achieve the system functionality in such thin and flexible packages, it is necessary to integrate power and data telemetry, signal conditioning and wireless communication functions with thin, flexible and embedded components. High-density circuitry with advanced component integration in flexible substrates is the gateway to realize this technology evolution.

Neural recording systems sense and communicate action or field potentials for several purposes such as identification of pathological conditions, motor intent, and drive a feedback therapeutic or functional neurostimulation system. They are fabricated at different scales depending on the electrode or channel count for the intended function, data rates or bandwidth, and power budget. For systems that record from 32, 64 or 100 channels, the data rates exceed $50 \mathrm{Mbps}$ and the power requirements exceed $100 \mathrm{~mW}$, leading to an energy consumption of $0.1-2 \mathrm{~nJ} / \mathrm{bit}$. One common way to design such wireless neural recording systems is to have them head-mount on the top of the skull. In this scenario, the rigid package with communication front-end, analog frontend signal modulation and battery is assembled on PCB substrates and encased in hermetic packages with feedthroughs that interface with electrodes. The size of the system can be as high as $100 \mathrm{cc}$. For example, a system of $51 \times 38 \times 38 \mathrm{~mm}^{3}$ was designed with a power consumption is $63.2 \mathrm{~mW}$, in which $28 \mathrm{~mW}$ is consumed by the $\mathrm{RF}$ transmitter[1]. A $1120 \mathrm{~mA}$-hr battery operates for 2.9 days 


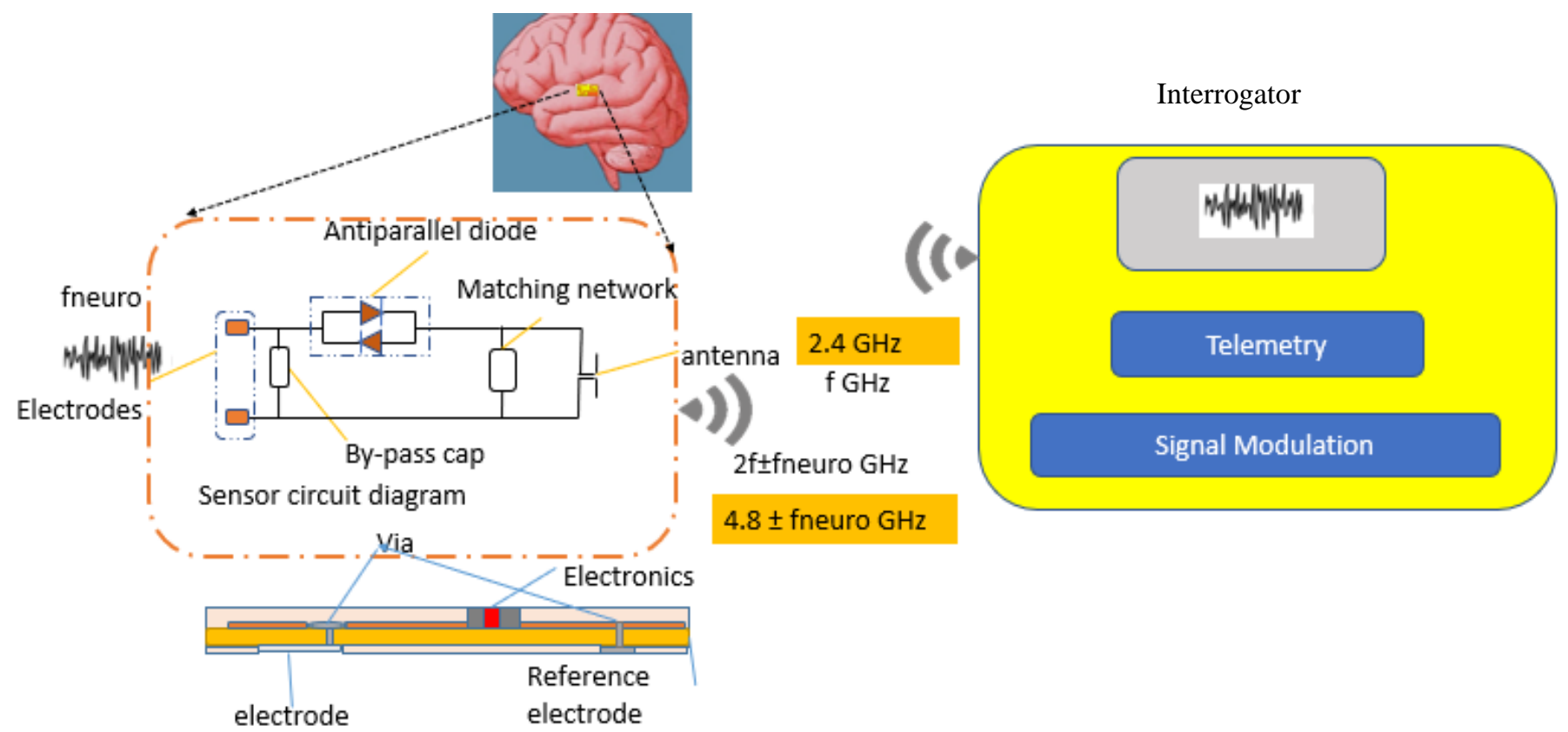

Fig. 1. Neural Recording System: Sensor circuit diagram with APDP, antenna and filter/bypass capacitor. Interrogating signal $2.4 \mathrm{GHz}$ is sent to the implanted sensor, $\mathrm{f}_{\text {neuro }}$ comes from the brain and the mixer circuit yields $\left(4.8 \mathrm{GHz} \pm \mathrm{f}_{\text {neuro }}\right)$ and received signal is sent back to external circuitry.

in this case. In order to avoid replacing battery, rechargeable battery with wireless telemetry is implemented. For example, a head-stage neural recording system with $56 \times 42$ $\times 9 \mathrm{~mm}^{3}$ was realized on a rigid package with rechargeable battery [2]. The preamplifier and controller consume about 6 $\mathrm{mW}$ and $1 \mathrm{~mW}$ respectively. Inductive charging becomes critical for communication with FSK (frequency shift key) that consumes $\sim 100 \mathrm{~mW}$ at Mbps data rates. When the battery is charging, the average power can increase to $100 \mathrm{~s}$ of $\mathrm{mW}$. Even though the system is powered by inductive coils, it has several issues. Overall power consumption is 350 $\mathrm{mW}$, which is high enough that it may negatively affect the tissue as it is well-documented that more than $40 \mathrm{~mW} / \mathrm{cm}^{2}$ power dissipation raises the tissue temperature by more than $2{ }^{\circ} \mathrm{C}$. Another way to design a neural recording system is in the form of subcutaneous microsystem. Such a system also has a recording unit and an external interrogator like the ones used in cortical recording. Implanted device has IC connected with probes, and the external device contains an antenna for data telemetry and inductive coil for power telemetry. Power consumption in such scenario is less than $30 \mathrm{~mW}$ [3]. Since battery has limited lifetime, researchers have pursued ultra-low-power integrated circuit (IC) technology to bypass the limited lifetime issue of battery and the associated safety and hazard issue. In such cases, customdesigned 32 channels, low power ICs, logic controller are used. Moreover, the whole system is on a rigid PCB, which is not mechanically compatible with the brain tissue and has higher probability to create inflammatory responses that prevent safe and long-term use [4]. To reduce power consumption, researchers implemented multichannel electrode array with a uniquely designed low-power IC that is co-packaged with microfabricated traces and electrodes on Parylene C. IC is powered by an inductive coil at $300 \mathrm{MHz}$. The total size of the sensor is $6.5 \mathrm{~mm} \times 6.5 \mathrm{~mm}$ and consumes $0.22 \mathrm{~mW}$ for 64 channels. Wireless communication is through backscattering. The packaged devices are assembled on a rigid PCB with solder to form the system interconnections. [5].

The other class of neural recording systems comprise of distributed individual untethered units that record from a single or few sites These can be miniaturized to mm-scale unlike the other units that are of 10-100 cc, and are also referred to as recording nodes or motes. These nodes are interrogated through new innovative transduction mechanisms and provide several unique opportunities for simplifying neural recording. Examples of such transduction include ultrasonic, RF, magnetoelectric or multiferroic excitations. These multiphysics-based transduction techniques effectively reduce the communication module and power consumption. Ultrasonic energy is utilized to realize a small, tiny single channel neural recording device, which is sometimes referred to as neural dust. This device includes a piezoelectrical crystal and a transistor. An external transducer is used to communicate with the implanted neural dust. This technology has edge over the RF energy as ultrasound energy does not attenuate as much as $\mathrm{RF}$ energy in the tissue. The footprint of the sensor is $0.8 \mathrm{~mm}$ $\times 3 \mathrm{~mm} \times 1 \mathrm{~mm}$. However, a major drawback of the system is that ultrasound energy cannot penetrate efficiently through the skull [6]. RF backscattering technique is utilized to 
realize fully passive and implantable neural recording devices. In such systems, an antiparallel diode, a by-pass capacitor, and matching network are integrated together on a PCB substrate. The whole wireless neurosensor is designed to a size of $15 \mathrm{~mm} \times 16 \mathrm{~mm} \times 1.5 \mathrm{~mm}$. With further advances in design, the device is miniaturized to $10 \mathrm{~mm} \times 9 \mathrm{~mm} \times$ $2.2 \mathrm{~mm}$ [7], [8]. Existing neupotential sensors are based on rigid or flexible PCB substrates. In either scenario, bulky prep-packaged dies are used, and rigid soldering or anisotropic conductive paste (ACP) are implemented to interconnect the package and dies. Therefore, current sensors are based on thick modules.

In this study, a miniaturized flexible single layer passive neuropotential sensor $(9.5 \mathrm{~mm} \times 8.7 \mathrm{~mm} \times 0.5 \mathrm{~mm})$ is demonstrated. Liquid Crystal Polymer (LCP) substrate is used as the flexible carrier for the circuit. A miniaturized spiral planar antenna is co-integrated with antiparallel diode mixer and a by-pass capacitor on the thin-flex LCP substrate with heterogeneous integration. The entire sensor is covered with biocompatible PDMS, leading to thin and flexible neural recording units for potential use in both onskin and implantable applications.
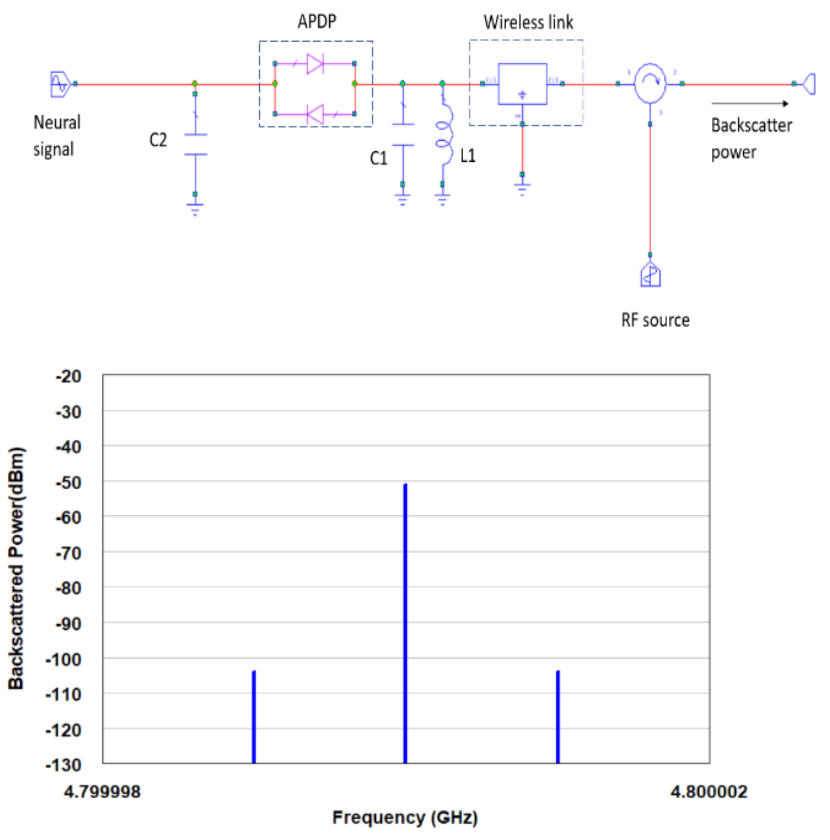

Fig. 2. Complete circuit model of neuro sensor (top), Backscattered sideband power shown in the frequency domain. The predicted power level is $-104 \mathrm{dBm}$ (bottom)

\section{NEUROSENSOR CIRCUIT SIMULATION AND FABRICATION}

Neural recording system comprises of two subsystems: 1) Implanted sensor, and 2) Interrogator, as shown in Fig. 1. The external device or interrogator transmits a wireless 2.4 $\mathrm{GHz}$ carrier to the implanted transponder. After receiving the carrier signal, implanted sensor mixes the $2.4 \mathrm{GHz}$ signal with low-frequency neural signals to produce the second harmonic at $4.8 \mathrm{GHz} \pm \mathrm{f}_{\text {neuro }}$. The modulated signal is then sent back to the external device or interrogator. Eventually, $4.8 \mathrm{GHz} \pm \mathrm{f}_{\text {neuro }}$ can be observed or viewed at the spectrum analyzer (frequency domain) or demodulated to the original time-domain signal that represents $f_{\text {neuro }}$. The implanted sensor (or neural recorder) comprises of two components: 1) Passive Mixer circuit 2) Wireless telemetry. An RF antenna operating at $2.4 \mathrm{GHz}$ and $4.8 \mathrm{GHz}$ acts as the wireless telemetry interface, and the mixer modulates neural signal $\left(f_{\text {neuro }}\right)$ and backscatters $\left(4.8 \mathrm{GHz} \pm \mathrm{f}_{\text {neuro }}\right)$ to the interrogator or an external device via the antenna in the implanted sensor.

One of the challenges in implanted neurosensors is to decrease the antenna size to achieve minimally invasiveness. Several miniaturization techniques have been developed. These include 1) Stacking multiple radiating patches on top of each other 2) Planar inverted F antenna configuration 3) Capacitive, inductive, or split-ring loading to match impedance 4) Increasing current path by meandering or spiraling 5) Implementing antennas on substrates with high dielectric constant. Although these techniques document antennas with small footprint and good radiation performance, higher thickness has always been a major drawback. Since our application requires thinness, flexibility, and biocompatibility, these telemetries fall short in meeting the requirements. Recent work reported antennas on flexible substrates with low dielectric constant. However, their footprints are generally larger. To remove the substrate thickness, various coplanar antenna topologies are also investigated. In our work, to meet the flexibility, stability and thinness requirements along with resistance to moisture, we chose LCP with a coplanar spiral structure to shrink the antenna size. Such a structure was first shown in[9], however, with a single band. Our approach advances this further to achieve a broad dual-band $(2.4 \mathrm{GHz}$ and $4.8 \mathrm{GHz})$ performance.

This antenna-in-package approach also co-integrates the antenna with passive circuitry to realize a miniaturized neural sensor. The neural sensor's schematic circuit is shown in Fig. 1. The planar antenna was designed on Rogers $3850 \mathrm{HT}$ substrates with permittivity of 2.9 and loss tangent of 0.002 . A subharmonic mixer is operated with a local oscillator (LO) at half of the typical conventional mixer's LO frequency. It yields the $2 \mathrm{f}_{\mathrm{LO}} \pm \mathrm{f}_{\text {Neuro }}$ signal with $\mathrm{f}_{\mathrm{LO}}$ while conventional mixers generate the same output with $2 \mathrm{f}_{\mathrm{LO}}$. The subharmonic mixer circuit's components are an antiparallel diode pair, a matching circuit, a bypass capacitor, and a pair of electrodes. An antiparallel diode pair (APDP) (Si MA4E2508L-1112) and matching network with transmission line and discrete bypass capacitors $(7.5 \mathrm{pF})$ were integrated with the antenna to accomplish the complete design of the neural sensor 


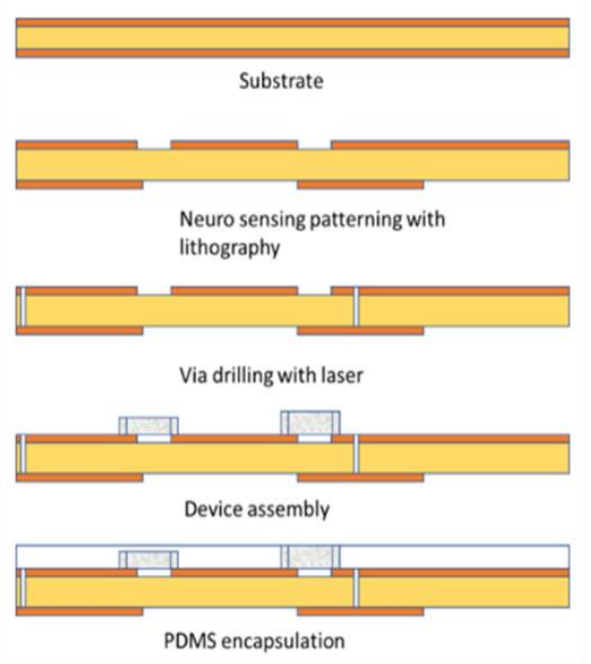

a)

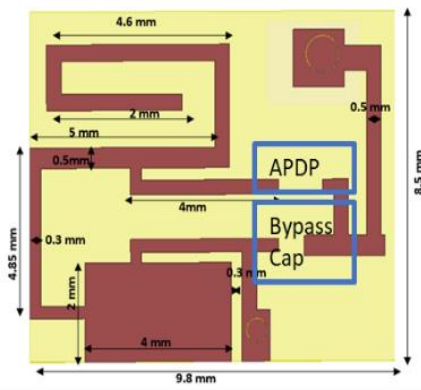

b) c)

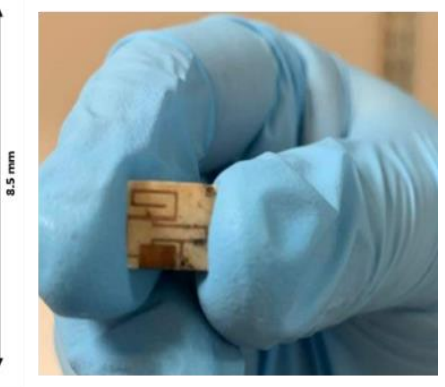

Fig. 3 a) Device fabrication process and assembly b) Geometry of the neurosensor and with electronics assigned in the sensor c) optical photo of the fabricated neuro sensor

Estimation of the overall system performance metrics such as total end-to-end chain loss and backscattered power with the passive circuit that comprises of an external antenna and implanted antenna, diode, and capacitor integration requires detailed circuit simulations. Cadence ${ }^{\circledR}$ AWR Design Environment ${ }^{\circledR}$ was used to simulate the whole neurosensor and predict the backscattered power. Matching network is required to match the antenna and the APDP impedance. The role of bypass capacitor is to shunt the $2.4 \mathrm{GHz}$ wireless link. APDP performance metrics from vendor datasheets were input into the model. An AC signal source at an amplitude of $1 \mathrm{mV}_{\mathrm{pp}}$ and frequency of $1 \mathrm{kHz}$ ( $f_{\text {neuro }}$ ) was used to mimic the neuro potential. For an input power of $15 \mathrm{dBm}$, the backscattered signal $(4.8 \mathrm{GHz} \pm 1 \mathrm{kHz})$ power is approximately $-105 \mathrm{dBm}$ for the modulated neural signal, which is well above the sensitivity of the signal analyzer $120 \mathrm{dBm}$. The simulated circuit performance with sideband performance is shown in Fig. 2. To detect the neuro signal, the backscattered power has to be $5 \mathrm{~dB}$ above the signal analyzer sensitivity.
As described before, Rogers 3850 HT was used as the substrate because of its excellent properties at high frequencies, good dimensional stability and low moisture absorption rate. The fabrication process is illustrated in Fig. 3. Subtractive copper patterning with dry-film photoresist sensors was performed to acquire the desired line definition and high conductivity for sensor traces in contrast to printed silver traces. Dry-film photoresist (Hitachi Chemicals, RY$5115 \circledR)$ was utilized to eventually support large-area fabrication at low cost. The process starts with substrate cleaning and microetching to enhance adhesion during photoresist patterning and copper etching. UV light was used for lithography at $360 \mathrm{~nm}$ narrowband wavelength with an energy dose of $100-120 \mathrm{~mJ} / \mathrm{cm}^{2}$. The photoresist pattern for the copper trace circuit was then developed in a $1 \%$ sodium borate solution at $60^{\circ} \mathrm{c}$ for 65 seconds, followed by rinsing in DI water. In order to improve the etching rate, the etchant was heated to $60{ }^{\circ} \mathrm{C}$. At the end, photoresist was removed with acetone to expose the copper traces. Substrate was subsequently laser-drilled with vias to interface with the electrodes on the backside. Silver loading with ink content of $65 \%$ was used to fill the vias with a vacuum-assisted viafilling machine. The via electrical connectivity was tested with a multimeter. Compared to silver pastes with $85 \%$ particle loading, lower loading of $65 \%$ showed better mechanical strength and lower contact resistance in our previous work. Conductive silver elastomer adhesive as chip-to-flex interconnection layers was utilized for surface assembly of devices to enhance electrical and reliability performance. For biocompatibility, the whole sensor was encapsulated with a PDMS coating.

\section{RESULT AND DISCUSSION}

Electrical Characterization Results: The measurement set-up for evaluating the prototype is shown in Fig. 4. A local oscillator signal was generated by a signal generator. The transmitted power was set at $15 \mathrm{dBm}$, which is well below the APDP's absolute maximum $(20 \mathrm{dBm})$ and provided to the external interrogating antenna through a circulator. An arbitrary function generator was used for creating the emulated neurosignal $f_{\text {neuro }}=1 \mathrm{kHz}$ with a magnitude of $1 \mathrm{mV}_{\mathrm{pp}}$. The neurorecording device was positioned inside the brain phantom. The phantom consists of skin, bone, dura/gray, and white matter. Recipe of the phantom was taken from prior work [7]. Spectrum analyzer was used to detect the received signal $\left(4.8 \mathrm{GHz} \pm \mathrm{f}_{\text {neuro }}\right)$ at the reader side, as shown in Fig. 4. Measured spectral response is also shown in the figure. For an input power of $15 \mathrm{dBm}$, the backscattered sideband RF power is measured as $-109 \mathrm{dBm}$ while the emulated neural signal at $1 \mathrm{mV}_{\mathrm{PP}}$ or $-58 \mathrm{dBm}$. Overall system loss was around $50 \mathrm{~dB}$ which is reasonable to detect neural signal $1 \mathrm{mVpp}$ at $1 \mathrm{kHz}$ while implantable and external antenna is $17 \mathrm{~mm}$ away, as illustrated by Fig. 4 b). The discrepancy between the 
measured value of $-109 \mathrm{dBm}$ and the simulated value of the sideband power of $-105 \mathrm{dBm}$ in Fig. 2 is $4 \mathrm{~dB}$. This

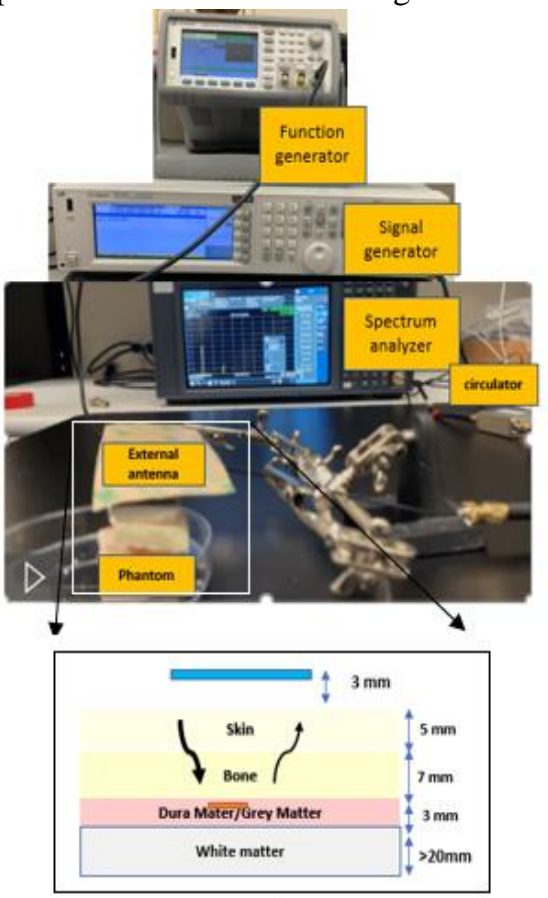

a)

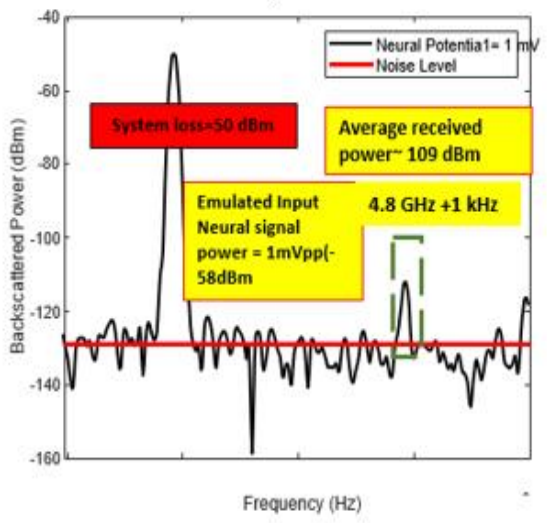

b)

Fig. 4. a) Experimental setup for neuro sensor b) Frequency domain performance of the sensor. Emulated neural voltage is $1 \mathrm{mVpp}$ and frequency is $1 \mathrm{kHz}$. Average received power is $108 \mathrm{dBm}$. External antenna and sensor are $17 \mathrm{~mm}$ away in this case.

difference between simulation and measurement results is mainly because of fabrication and additional unaccounted parasitic values. When implantable neural sensors are placed under the skin, and interrogator is $2 \mathrm{~mm}$ away, the system loss is approximately $30 \mathrm{~dB}$, deduced as the difference between the input neural power of $-58 \mathrm{dBm}$ and the received sideband power of $-89 \mathrm{dBm}$, as illustrated in Fig. 6., which is similar to the values reported in [8].System Performance Analysis and Projection: The key performance metrics for a neural recording unit are a) low interrogating power, b) high backscattering power because of low conversion, propagation and other system losses and c) high sensitivity to weak (20 microvolts) neurosignals. These three aspects are examined in detail here with a simple diagram Fig. 7 (Top)

The Path 1 loss is guided by two factors. Propagation loss at $2.4 \mathrm{GHz}$ and matching loss. The incoming power should compensate for the propagation losses from the interrogator to the device through the air and tissue, and losses from mismatch.

Path1 Loss $=L_{\text {Propag@2.4 GHz }}[d B]+L_{\text {Match }}[d B]$

We will focus on Path 2 loss, which is also interpreted as system loss in this work because it results in lower recorded neuropotential signal power. The System loss in Path 2 is related to the conversion loss, matching and propagation losses, as shown in Fig. 7 (top) and described in Eqn (2).

System loss can be represented as System loss in Path 2[dB]

$$
\begin{aligned}
& =L_{\text {Conversion loss } @ 4.8 \mathrm{GHz} \pm \text { fneuro }}[\mathrm{dB}] \\
& +L_{\text {Propag@ } @ 4.8 \mathrm{GHz} \pm \text { fneuro }}[\mathrm{dB}] \\
& +L_{\text {Match }}[\mathrm{dB}]
\end{aligned}
$$

$\mathrm{L}_{\text {Conversion losses }}$ is the loss during the conversion from input carrier power to the backscattered carrier signal power, $L_{\text {Propag@4.8GHz fneuro }}$ is the propagation loss at $4.8 \mathrm{GHz}$ $\pm \mathrm{f}_{\text {neuro }}$ and $\mathrm{L}_{\text {Match }}$ is the mismatch loss between implanted antenna and APDP. The conversion loss is lowest (9dBm) when the input power is $-2 \mathrm{dBm}$. As the power deviates from the optimal value, the losses increase. The plot of the conversion loss as a function of the incoming power was simulated and estimated, as shown in Fig. 5., for the completion of the discussion.

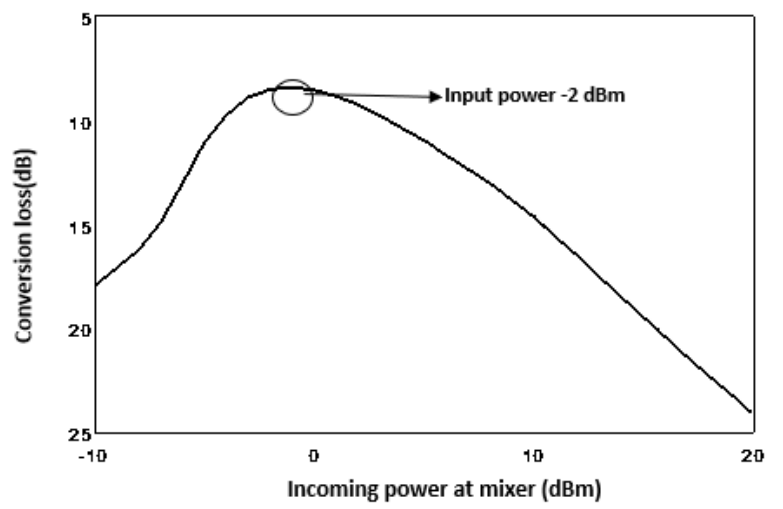

Fig. 5. Incoming Power at mixer vs Conversion loss. Optimized input power is $-2 \mathrm{dBm}$ and corresponding conversion loss is $9 \mathrm{dBm}$

Tissue behaves as a conductor at high frequencies while human body acts as a high-dielectric loss medium at high frequencies since real part of the permittivity begins to dominate the conductivity. RF signal propagates through a high-dielectric-constant medium to a low-dielectricconstant medium, at it bends away from the direction 
perpendicular to the interface. In other words, signal effectively travels longer distances in the tissue and encounters multiple reflections. Therefore, placing the implantable neural sensor under the skin would increase the propagation loss and leads to lower power carried by the incoming carrier at $2.4 \mathrm{GHz}$. With higher propagation loss, the transmitter needs to transmit at higher power to keep the conversion loss under control so that higher signal sensitivity is achieved. One other way to avoid this high propagation loss is to introduce multiferroic antennas[10] that operate at lower frequencies.

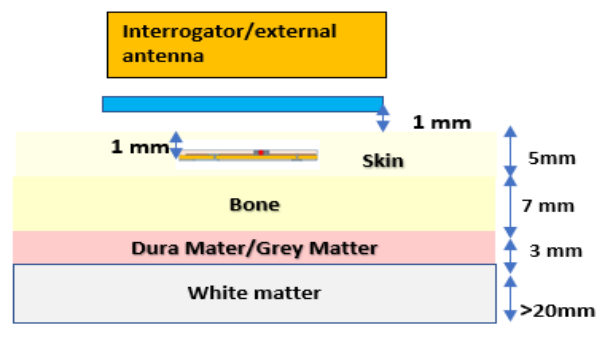

a)

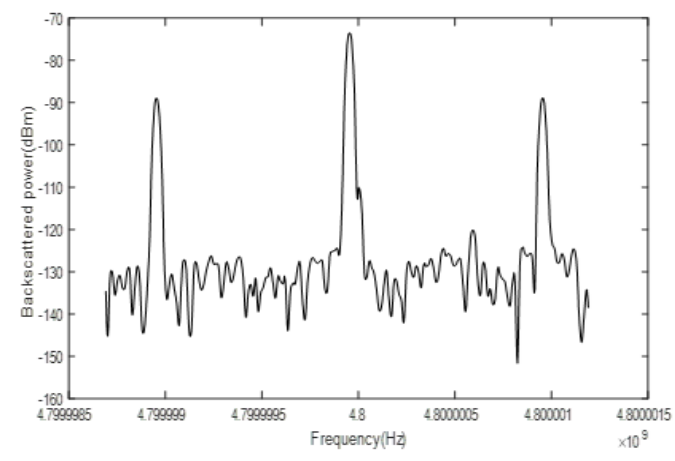

b)

Fig. 6. a) Position of the interrogator antenna from implantable neural recording sensor. It is $2 \mathrm{~mm}$ away in this specific case. b) Frequencydomain spectrum of the backscattered power is at around $-89 \mathrm{dBm}$

The key system metric is the sideband neural signal power that can be detected by the receiver circuit. Lowest neural signal detection is dependent on minimum receiver sensitivity and system loss. With low system loss, we can record weakest or lowest neuropotential signal. Subtracting the lowest detectable neural signal power from minimum receiver sensitivity yields the tolerable system loss, as described by Eqn (3)

\section{Lowest Detectable Neural Signal = Lowest Neuropotential $[\mathrm{dBm}]$ $=$ Minimum of the receiver Sensitivity + System loss in Path 2

From the analysis, the backscattered power should be atleast $-125 \mathrm{dBm}$ so that the carrier power at the receiver is adequate and above $-130 \mathrm{dBm}$. For this receiver sensitivity of $-130 \mathrm{dBm}$, the lowest neuropotential signal is $-90 \mathrm{dBm}$, when the target system loss $40 \mathrm{~dB}$. In other words, as long as the sum of conversion modulation loss, matching loss and propagation loss at $4.8 \mathrm{GHz}$ are less than $40 \mathrm{~dB}$, we can detect neuropotential signals as low as 20 microvolts. From our analysis, we can also infer that reducing distance leads to decrease in propagation loss. When fixing the distance between external antenna and implantable sensor to $2 \mathrm{~mm}$, we predict that $20 \mu \mathrm{v}$ can be acquired with the optimal incoming power at $2.4 \mathrm{GHz}$ power.
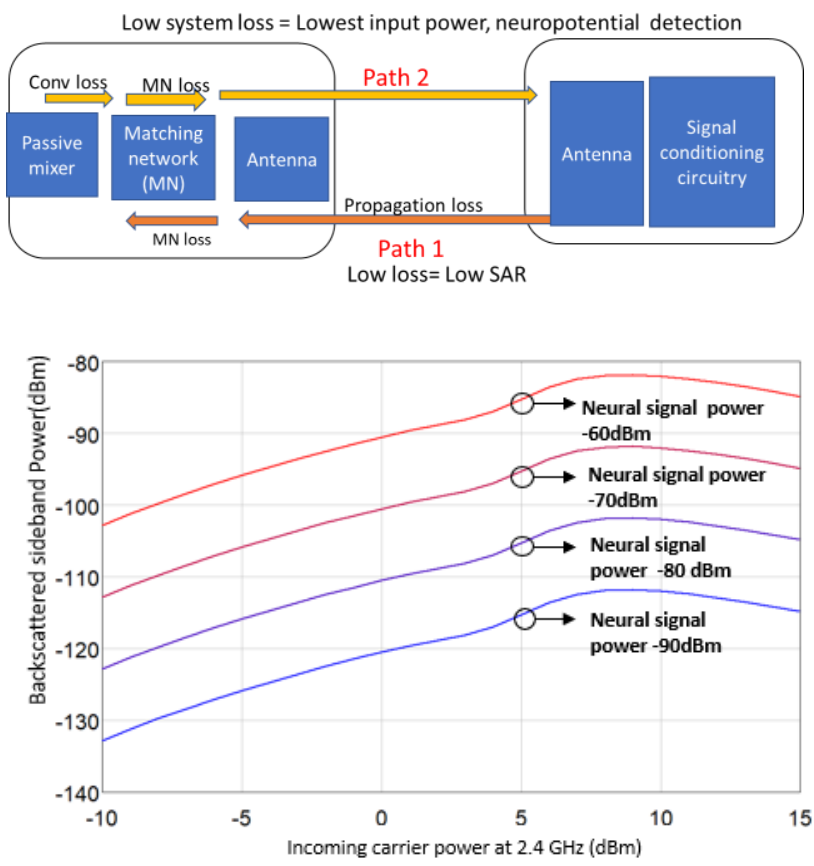

Fig. 7. System loss includes conversion loss, matching network loss and propagation loss (Top). Backscattered sideband power as a function of the power of the incoming carrier $(2.4 \mathrm{GHz})$ power at different neural signal power (Bottom)

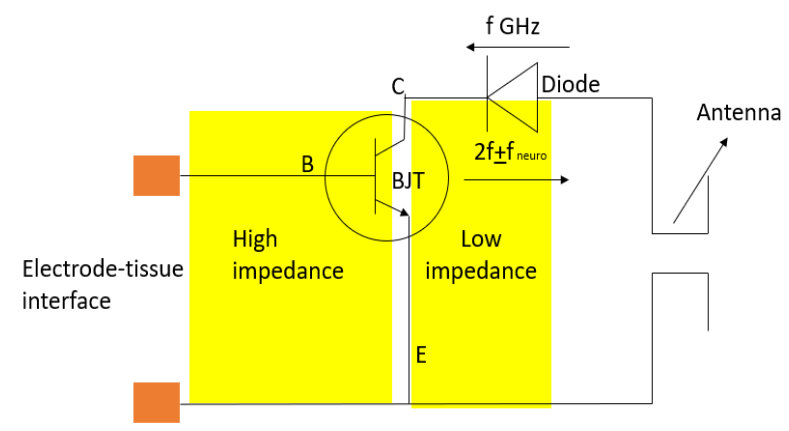

Fig. 8. A novel passive neural recording sensor with a simple BJT and Diode. BJT changes the high impedance into low impedance

As seen from the equation, it is critical to minimize both the propagation and mixing losses to achieve superior signal sensitivity. The mixing losses arise from the APDP and also the impedance mismatch between the incoming neural signal and the APDP. For example, the electrodes are at high impedance, which invariably lead to substantial signal losses 
that the APDP receives at its input. Impedance matched recording is hence preferred to lower these losses. One way to achieve this is by utilizing electrodes with higher surface area and closer to the neural signal source. Impedance transformation with a Bipolar Junction Transistor (BJT) device is also an alternative approach to address this challenge [6]. We will only briefly discuss novel circuit schematic here in the Fig. 8. Diode not only acts as a DC bias for BJT but also as a mixer, while BJT converts high impedance coming from electrode-tissue interface into low impedance, resulting in recording low-potential brain signal [11]. However, small form factor with bare die NpN BJT and diode with embedded packaging are yet to be realized.

Specific Absorption Rate (SAR) is the measurement of RF energy absorbed in human body or tissue while a wireless transmission occurs. The SAR generated by our neurosensing system was assessed by ANSYS ${ }^{\circledR}$ HFSS. The implant was $1 \mathrm{~mm}$ below the skin layer and external antenna was $1 \mathrm{~mm}$ above the skin layer, as shown in Fig. (5a). An RF input power of $5 \mathrm{dBm}$ at $2.4 \mathrm{GHz}$. The calculated $\mathrm{SAR}_{\mathrm{gg}}$ is $0.97 \mathrm{~W} / \mathrm{kg}$ while $\mathrm{SAR}_{10 \mathrm{~g}}$ is $0.13 \mathrm{~W} / \mathrm{kg}$, which are in compliance with Federal Communications Commission (FCC) for uncontrolled environment exposure $\left(\mathrm{SAR}_{1 \mathrm{~g}}<1.6 \mathrm{~W} / \mathrm{kg}[12]\right.$ and International Commission on Non-Ionizing Radiation Protection (ICNIRP)(SAR $\left.{ }_{10 \mathrm{~g}}<2 \mathrm{~W} / \mathrm{kg}\right)[13]$ respectively. Therefore, our neurosesning system is projected to meet the patient safety guidelines.

Package Size Analysis: The package size is determined by the antenna footprint, mixer and matching components and the interconnects. This size is usually much smaller than the electrode extensions between the neurosensor and the neural interfaces. Since the electrode designs are customized to the

TABLE I

Size and packaging analysis and comparison of neural recording devices with our work

\begin{tabular}{|l|l|l|}
\hline [1] & $51 \times 38 \times 38 \mathrm{~mm}^{3}$ & $\begin{array}{l}\text { Rigid substrate, thick } \\
\text { prepacked die and } \\
\text { rigid soldering for } \\
\text { interconnections }\end{array}$ \\
\hline [2] & $56 \times 42 \times 9 \mathrm{~mm}^{3}$ & \\
\hline$[\mathbf{7 , 8}]$ & $\begin{array}{l}10 \mathrm{~mm} \times 9 \mathrm{~mm} \times 2.2 \\
\mathrm{~mm}\end{array}$ & $\begin{array}{l}\text { Flexible substrate, } \\
\text { thin chip and silver } \\
\text { elastomer for } \\
\text { interconnection }\end{array}$ \\
\hline $\begin{array}{l}\text { Our } \\
\text { work }\end{array}$ & \begin{tabular}{l}
$9.5 \mathrm{~mm} \times 8.7 \mathrm{~mm}$ \\
\hline
\end{tabular}
\end{tabular}

target recording application and is not a key focus of this work, we only compare the neurosensor size without including the electrode size. The size or form-factor of previous rigid packages on circuit boards with current packages in this work are compared in Table 1. This work implements planar antennas on flexible substrates. The proposed monopole spiral antenna is agnostic to the substrate thickness, making it much thinner than today's neurosensor devices. As the telemetry is substrate thicknessindependent, the neural recording system can be implemented on much thinner substrates (for example, $50 \mu \mathrm{m}$ thick flexible substrate). Comparing to other published work, our work thus demonstrates thinner, smaller and flexible packages due to planar antenna design, single layer circuitry, and thin chips.

The next key factor that determines the module thickness is the component thickness and off-chip interconnection height. In traditional packaging, electronic components are assembled on the substrate with thick solder joints at temperatures of above $230^{\circ} \mathrm{C}$ and encapsulated, which eventually lead to thicker packages. Moreover, prepackaged thicker chips in leadframe QFN packages contribute to bulky packages. To aim for thinner substrates, it is necessary to embed electronic components into the flexible substrate. This is consistent with the emerging trend of sub-100 micron dies for embedding and fan-out packaging technologies. In fan-out packaging with chip-embedding in flexible carriers, substrates with cavities and a back-up layer are fabricated as the first step. Devices are then assembled into the cavities and off-chip interconnects are directly formed from the chip pads to the package traces with printed conductive elastomers. Additionally, thinner die is connected with copper traces and silver-elastomer interconnects, which barely adds any height to the package. More importantly, advanced packaging with embedding dies and thin-film passive components in flex will further reduce the size of neurosensor [14]. Such trends have been recently realized to further reduce the package form-factor. In our future work, passive impedance matching neural recording sensor will be realized with embedded packaging in miniaturized form.

\section{SUMMARY}

Heterogeneous integration of thin active devices, power and data telemetry, and storage components in thin, flexible substrates will pave the way for miniaturized bioelectronics. Towards this goal, we have shown a passive telemetry communication system for transmitting neural signals. Simplified passive backscattering approach miniaturizes the topology and bill-of-materials for system components. Innovative topologies with monopole spiral miniaturized antennas further reduced the system size. Complete system loss analysis showed the propagation losses of approximately $30 \mathrm{~dB}$ either way - for the incoming and backscattered signals. The projected signal sensitivity for this system is $20 \mu \mathrm{v}$, assuming a receiver sensitivity of -130 $\mathrm{dBm}$. With decreasing distance between external antenna and implantable, input power is reduced to $5 \mathrm{dBm}$, which is adequate to acquire neuropotential as low as $20 \mu \mathrm{v}$. The 
lowest input power for the system function can be further reduced with advances in the diode or mixers.

\section{REFERENCES}

[1] C. A. Chestek, V. Gilja, P. Nuyujukian, R. J. Kier, F. Solzbacher, S. I. Ryu, et al., "HermesC: lowpower wireless neural recording system for freely moving primates," IEEE Transactions on Neural Systems and Rehabilitation Engineering, vol. 17, pp. 330-338, 2009.

[2] D. A. Borton, M. Yin, J. Aceros, and A. Nurmikko, "An implantable wireless neural interface for recording cortical circuit dynamics in moving primates," Journal of neural engineering, vol. 10, p. 026010, 2013.

[3] K. W. Cheng et al., "100-Channel wireless neural recording system with $54-\mathrm{Mb} / \mathrm{s}$ data link and $40 \%$ efficiency power link," Proceedings - 2012 IEEE Asian Solid-State Circuits Conference, A-SSCC, pp. 185-188, 2012, doi: 10.1109/IPEC.2012.6522656.

[4] C. S. Mestais, G. Charvet, F. Sauter-Starace, M. Foerster, D. Ratel, and A. L. Benabid, "WIMAGINE: wireless 64-channel ECoG recording implant for long term clinical applications," IEEE transactions on neural systems and rehabilitation engineering, vol. 23, pp. 10-21, 2014.

[5] R. Muller et al., "A Minimally Invasive 64Channel Wireless $\mu \mathrm{eCoG}$ Implant," IEEE Journal of Solid-State Circuits, vol. 50, no. 1, pp. 344-359, Jan. 2015, doi: 10.1109/JSSC.2014.2364824.

[6] D. Seo, R. M. Neely, K. Shen, U. Singhal, E. Alon, J. M. Rabaey, et al., "Wireless recording in the peripheral nervous system with ultrasonic neural dust," Neuron, vol. 91, pp. 529-539, 2016.

[7] A. Kiourti, C. W. L. Lee, J. Chae, and J. L. Volakis, "A wireless fully passive neural recording device for unobtrusive neuropotential monitoring," IEEE Transactions on Biomedical Engineering, vol. 63, no. 1, pp. 131-137, Jan. 2016, doi: 10.1109/TBME.2015.2458583.

[8] C. Moncion, L. Balachandar, S. BojjaVenkatakrishnan, J. J. Riera, and J. L. Volakis, "Fully-Passive Wireless Implant for Neuropotential Acquisition: An in Vivo Validation," IEEE Journal of Electromagnetics, $R F$ and Microwaves in Medicine and Biology, vol. 3, no. 3, pp. 199-205, Sep. 2019, doi: 10.1109/JERM.2019.2895657.
[9] M. W. A. Khan, E. Moradi, L. Sydänheimo, T. Björninen, Y. Rahmat-Samii, and L. Ukkonen, "Miniature coplanar implantable antenna on thin and flexible platform for fully wireless intracranial pressure monitoring system," International Journal of Antennas and Propagation, vol. 2017, 2017, doi: $10.1155 / 2017 / 9161083$.

[10] M. Zaeimbashi et al., "NanoNeuroRFID: A low loss brain implantable device based on magnetoelectric antenna," in IMBioc 2018 - 2018 IEEE/MTT-S International Microwave Biomedical Conference, Aug. 2018, pp. 205-207. doi: 10.1109/IMBIOC.2018.8428892.

[11] W. C. Chen, K. Guido, and A. Kiourti, "Passive impedance matching for implanted brain-electrode interfaces," IEEE Journal of Electromagnetics, RF and Microwaves in Medicine and Biology, vol. 3, no. 4, pp. 233-239, Dec. 2019, doi: 10.1109/JERM.2019.2904024.

[12] R. E. Fields, "Evaluating compliance with FCC guidelines for human exposure to radiofrequency electromagnetic fields," OET bulletin, vol. 65, 1997.

[13] A. Ahlbom, U. Bergqvist, J. Bernhardt, J. Cesarini, M. Grandolfo, M. Hietanen, et al., "Guidelines for limiting exposure to time-varying electric, magnetic, and electromagnetic fields (up to 300 GHz)," Health physics, vol. 74, pp. 494-521, 1998.

[14] S. Y. B. Sayeed, D. Wilding, J. S. Camara, D. Vital, S. Bhardwaj, and P. M. Raj, "Deformable Interconnects with Embedded Devices in Flexible Fan-Out Packages," International Symposium on Microelectronics, vol. 2019, no. 1, pp. $000163-$ 000168, Oct. 2019, doi: 10.4071/2380-45052019.1.000163. 